\section{Kompass \\ Dermatologie}

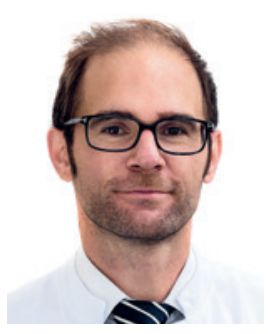

\section{Andreas Körber}

Gemeinschaftspraxis Dr. Cesko \&

Prof. Dr. Körber, Essen, Deutschland

Liebe Leserinnen und Leser, ein unerwartet aufregendes Jahr 2020 liegt hinter uns. Sicherlich hat niemand vor einem Jahr vorhersehen können, wie dramatisch und umfangreich das Thema COVID-19 unseren privaten und beruflichen Alltag bestimmen wird. Und es wird uns auch im Jahr 2021 über weite Strecken begleiten. Selten hat etwas derart umfassende und schnell umzusetzende Änderungen bedingt, wie diese Pandemie. Seien es neue Hygienekonzepte in Klinik und Praxis, oder die notwendige Flexibilität, sich auf Lockdowns oder positive Testergebnisse einzustellen. Wir haben uns an virtuelle Fortbildungen gewöhnt (oder gewöhnen müssen) und festgestellt, dass dies eine sehr gute Option darstellt, jedoch auch, dass das wirkliche Zusammenkommen schöner ist. Des Weiteren brachte die Pandemie mit sich, dass alternative Konzepte in vielen Bereichen entwickelt wurden. Es gab enorme Veränderungen insbesondere in Bezug auf digitale Lösungen, wie beispielsweise die Videosprechstunde oder store and foreward Systemen wie dem OnlineDoctor. Als hätten wir es bei der Themenplanung für 2020 geahnt, war die Digitalisierung in der Dermatologie auch der Schwerpunkt unserer letzten Ausgabe 2020.

\section{Was kommt nun 2021?}

Auch für das neue Jahr haben wir einen hochspannenden Rahmen für unsere einzelnen Ausgaben entwickeln können. Be-

\title{
Start frei für 2021
}

ginnen wird es mit einem stets aktuellen Themenkomplex, den entzündlichen Hauterkrankungen. Neben der Psoriasis vulgaris und ihrer Komorbidität, spielt die Musik im Sinne von innovativen neuen Therapieoptionen zunehmend bei der atopischen Dermatitis. Hier werden in den kommenden 2-3 Jahren zahlreiche neue Möglichkeiten zur systemischen Therapie hinzukommen. Das Prozedere, auch in Bezug auf therapeutische Implikationen bzw. Komorbidität, wird bei beiden Erkrankungen zunehmend verbessert und findet Einzug in regelhaft aktualisierte Leitlinien und Behandlungspfade, welche die Vorlage für die praktische Umsetzung darstellen. Auch hier gab es in 2020 Aktualisierungen der deutschen Neurodermitis Leitlinie und der europäischen Psoriasis Leitlinie (EDF). Zudem wurde Ende 2020 erstmalig neben dem bekannten Psoriasis-Behandlungspfad ein Behandlungspfad für die atopische Dermatitis vorgestellt.

Ich kann nur jedem von Ihnen ans Herz legen, sich diese aktuellen wie auch gut geordneten Manuskripte anzusehen, um jedem Patienten eine hocheffektive und individualisierte dermatologische Systemtherapie anbieten zu können. Neben den genuinen Erkrankungen werfen wir weiterhin natürlich auch hier einen Blick auf die Bedeutung von COVID-19 in Bezug auf entzündliche Dermatosen.

In den weiteren Ausgaben 2021 haben wir uns für klinisch immer wieder herausfordernde Themen wie die Umwelt- und Berufsder- matologie und die pädiatrischen Dermatologie entschieden, und auch hierfür renommierte Gastherausgeber gewinnen können. In der pädiatrischen Dermatologie nimmt, neben den klassischen pädiatrischen Fragestellungen, der Anteil von jungen Patienten zu, die beispielsweise systemische Therapien benötigen. Zudem wächst das therapeutische Arsenal für eine Vielzahl von pädiatrischen Erkrankungen.

Abgerundet wird diese Auswahl mit der Dermato-Onkologie, einer der Säulen unseres Fachs, bei der es zunehmend komplexer wird, ab einem gewissen Tumorstadium außerhalb von Hautkrebszentren adäquat zu behandeln.

Ich danke in diesem Rahmen von ganzem Herzen dem gesamten Team des Kompass Dermatologie sowie insbesondere den Gasteditoren und dem wissenschaftlichen Beirat für die große Unterstützung und die immer wieder tollen Artikel, die diese Zeitschrift immer hochaktuell und verständlich machen.

Bleiben sie alle gesund, ich hoffe wir sehen uns in 2021 dann auch mal auf nicht virtuellen Kongressen wieder.

Mit herzlichen Grüßen, Ihr

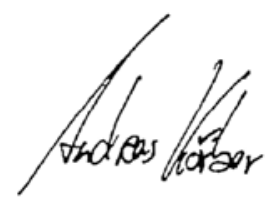

Andreas Körber information@karger.com @ (c) 2021 S. Karger GmbH, Freiburg www.karger.com/kkd

Karger'?
Prof. Dr. Andreas Körber

Gemeinschaftspraxis Dr. Cesko \& Prof. Dr. Körber

Rüttenscheider Str. 143

45147 Essen, Deutschland

Andreas.koerber@uk-essen.de 